\title{
Tinnitus: Characteristics, Need for Therapy, and Therapeutic Outcomes; Results of an International Patient Initiated Platform
}

\author{
Adriana L. Smit ${ }^{1,2 *}$, Markku Vesala ${ }^{3,4}$, Hazel Goedhart ${ }^{3,4}$, Job van Eijden ${ }^{1}$, \\ Christiaan Wempe ${ }^{1}$ and Inge Stegeman ${ }^{1,2,5,6}$ \\ ${ }^{1}$ Department of Otorhinolaryngology, Head and Neck Surgery, University Medical Centre Utrecht, Utrecht, Netherlands, \\ ${ }^{2}$ University Medical Center Utrecht Brain Center, University Medical Center Utrecht, Utrecht, Netherlands, ${ }^{3}$ Tinnitus Hub Ltd, \\ Amsterdam, Netherlands, ${ }^{4}$ Tinnitus Hub Ltd, London, United Kingdom, ${ }^{5}$ Department of Ophthalmology, University Medical \\ Center Utrecht, Utrecht, Netherlands, ${ }^{6}$ Epidemiology and Data Science, Amsterdam University Medical Centers, University \\ of Amsterdam, Amsterdam, Netherlands
}

OPEN ACCESS

Edited by:

Arianna Di Stadio,

University of Perugia, Italy

Reviewed by:

Elouise Koops,

University Medical Center Groningen, Netherlands

Pawel J. Jastreboff Emory University, United States

${ }^{*}$ Correspondence:

Adriana L. Smit a.l.smit-9@umcutrecht.n

Specialty section:

This article was submitted to

Neuro-Otology,

a section of the journal

Frontiers in Neurology

Received: 16 September 2021 Accepted: 20 December 2021

Published: 20 January 2022

Citation:

Smit AL, Vesala M, Goedhart $H$, Eijden JV, Wempe C and Stegeman I (2022) Tinnitus: Characteristics, Need

for Therapy, and Therapeutic Outcomes; Results of an International Patient Initiated Platform

Front. Neurol. 12:778450. doi: 10.3389/fneur.2021.778450
Introduction: So far, there is a gap of knowledge about factors influencing the impact of tinnitus, the need for treatment, as well as the experienced effect of regular and alternative tinnitus therapies. In this study, we analyzed the need for treatment and the outcomes of these treatments in an international patient initiated in tinnitus platform.

Materials and Methods: Two surveys were undertaken at an online tinnitus patient support community (www.tinnitustalk.com). The surveys were aimed at (1) using tinnitus treatment and outcomes and (2) the factors that influence tinnitus. Univariable logistic and linear regression were used to calculate the relation between the factors and the tinnitus impact as well as the relation with the used tinnitus treatments and the outcomes.

Results: Of the participants to the first survey ( $n=5,017), 2,914$ (58.1\%) used one or more tinnitus therapies, whereas others most commonly self-administered sound therapy $[n=1,562(31.1 \%)]$ and supplements/herbal medicines $[n=1,157$ (23.1\%)]. Being female [odds ratio (OR) 0.83 (95\% Cl 0.74-0.93, $p<0.01)$ ], tinnitus impact, and some degrees of hearing loss and hyperacusis were all statistically significantly associated with higher odds of having tinnitus treatment. Out of the second survey $(n=6,115)$, it was found that patient physical and psychological factors were statistically significantly related to tinnitus impact.

Conclusion: In this study, we demonstrated the usage and experience of (multiple) tinnitus therapy in patients. Several patient physical and psychological characteristics were found to be related to tinnitus impact and therapy usage. These outcomes might function as the next step to find a personalized treatment and to improve the tinnitus health care.

Keywords: tinnitus, therapy, treatment outcome, survey, patients 


\section{INTRODUCTION}

Tinnitus is the perception of sound without an external stimulus, often experienced as a ringing or buzzing sound $(1,2)$. It is a common condition with an approximate prevalence of 5.1$42.7 \%$, depending on the selected population and used definitions (3). While the underlying etiology of tinnitus is still debated, one hypothesis is that the tinnitus arises from changes in neural activity caused by reduced or lack of auditory input due to hearing loss which often accompanies tinnitus $(4,5)$. It is considered a complex condition, in which many components are responsible for perceived impact, such as loudness and tinnitusrelated difficulties such as sleep and concentration problems. The impact people experience is diverse and the individual needs of patients for tinnitus-related health care varies.

So far, several tinnitus treatment modalities are available. Due to the lack of curative treatments, these therapies all focus on relief from symptoms as the highest achievable goal. Of these, psychological therapy such as cognitive and/or behavioral therapy (CBT) $(4,6-8)$ or the application of hearing aids in case of accompanying hearing loss (4) is a part of the standard clinical care in many countries to improve the quality of life or reduce tinnitus-related distress (9-11). Considering the fact that there is no evidence for the effectiveness of drug treatments specifically for tinnitus (11), it is of interest that an estimation of 4 million offlabel prescriptions each year for tinnitus relief have been reported in Western Europe and the USA (3).

Due to the ongoing burden that people with chronic tinnitus are experiencing, some patients keep searching for a solution within regular and alternative therapies to solve their problem $(12,13)$. Thereby, there is a gap of knowledge about factors influencing peoples' tinnitus, the need for treatment, and the experienced effect of conventional and non-conventional tinnitus therapies. This is of special interest as people with tinnitus do not necessarily attend a physician in their search for tinnitus relief.

In order to improve the knowledge about a broad sample of individuals experiencing tinnitus, those with and those without burden, we assessed a worldwide internet-based cohort. We aim to analyse the factors of influence on the experienced tinnitus, the need for treatment, and the experienced effect of tinnitus therapy.

\section{MATERIALS AND METHODS}

\section{Ethical Considerations}

Ethical approval for this study and a waiver of informed consent were obtained from the local ethical committee of the University Medical Center Utrecht, Netherlands (local number: 19-134/C), for anonymous data collection and with the intention to use these data for scientific analyses. This study was performed in accordance with the Declaration of Helsinki (version 2013, Fortaleza) and the Medical Research Involving Human Subjects Acts (WMO).

\section{Study Design and Setting}

This study was conducted following a cross-sectional cohort design. By partnering with Tinnitus Hub to collect survey responses from members of their online patient support community, Tinnitus Talk (www.tinnitustalk.com), data of people experiencing tinnitus were retrieved. This platform was initiated in 2011 for worldwide peer-to-peer support services and is available for everyone without any costs and is written in English. Included in this study were two separate surveys conducted in February 2016 (Survey "Causes and Treatments") and October 2017 (Survey "Physical Links") on this platform.

\section{Study Aim}

The primary aim of this study was to analyse the characteristics of those individuals with tinnitus who underwent treatment and compare these characteristics to those who did not undergo treatment. Secondly, we aimed to evaluate the influence of different variables on experienced tinnitus.

\section{Participants}

Visitors of all ages of the website of the Tinnitus Talk platform were asked to fill out a questionnaire about their tinnitus and effect of treatments. Questionnaires were handled anonymously.

\section{Outcome Measures: Survey Causes and Treatments}

The online questionnaire of the survey "Causes and Treatments" was aimed at the factors that influence tinnitus and the experience with tinnitus treatment. It contained 32 items and included questions about demographics, tinnitus characteristics, cause of the tinnitus, comorbidities, tinnitus-related conditions, the impact of tinnitus on daily life, physiological/physical factors of influence on experienced tinnitus, received tinnitus treatment, and outcomes. Questions were checkboxes in which the participants had to select the answer most applicable to their situation (Attachment 1 Survey "Causes and Treatments").

Demographic variables included gender and age range. Tinnitus characteristics were scored with regard to tinnitus time of onset, pulsatile (described as tinnitus that is rhythmic), and somatic character (defined as that the tinnitus changes in volume by physical movement or touch). Comorbid conditions were asked for including hyperacusis (described as "a sensitivity to sounds, often you will think sounds are irritating and painfully loud when others hear them as normal") and hearing loss (categorized in "none known of," "mild hearing loss; may struggle a little to keep up with conversation," "moderate hearing loss; generally struggle to keep up with conversation," "severe hearing loss; often rely on lip reading as well as hearing"). The primary cause of tinnitus was scored according to the judgement of the participant, as well as if tinnitus caused any health-related conditions according to their opinion [e.g., stress, anxiety, depression, insomnia, panic attacks, depression, concentration problems, obsessive compulsive disorder (OCD-) type conditions, and substance abuse]. To score the effect of tinnitus on daily life, the awareness, bothersomeness, and frustration of the tinnitus over the last week was recorded on a scale from 1 to $10(1=$ not at all; $10=$ constantly). The effect of psychological (stress, anxiety, depression, panic attacks, and OCD-like conditions) and physical factors (jaw and neck problems, physical disabilities) were scored for their influence on 
the experienced tinnitus (made a lot better, made a little better, no difference, made a little worse, made a lot worse).

The participants were asked if they underwent any treatments for their tinnitus. If yes, items out of a long list of potential therapies were asked to be checked when applicable (consisting of pharmacological-, psychological-, and sound-therapy treatments and non-conventional therapies). Secondly, the effect of these therapies on tinnitus was rated by the participant (big improvement, small improvement, no change, made me slightly worse, made me a lot worse).

\section{Outcome Measures: Survey Physical Links}

The second questionnaire consisted of 22 questions and aimed at assessing the factors of influence on the experienced tinnitus. Questions were encompassing demographic variables, as well as the impact of tinnitus on daily life, tinnitus characteristics and cause, comorbid conditions (in particular, somatic factors), and the influence of factors on tinnitus (Attachment 2 Survey "Physical Links").

Demographic variables included gender and age in years. The impact of tinnitus was assessed as loudness and annoyance of tinnitus over the past week on a scale from 0 to 10 (loudness: $0=$ do not hear, $10=$ extremely loud/annoyance: $0=$ never annoyed, $10=$ constantly annoyed). The participants rated the last week awareness on a scale from 0 to $100(0=$ never aware, $100=$ always aware). The primary cause of the tinnitus according to the participant was scored. Tinnitus characteristics entailed the tinnitus duration (in years), pattern of perceived sounds during the day, and the effect of sounds on tinnitus. Hearing loss was scored by the question "do you have any hearing loss?" for which the answers were divided into categories as mentioned for survey "Treatment for tinnitus" with the addition of two categories: "hearing loss not diagnosed but I think so" and "not diagnosed but I don't think so."

Comorbid conditions and their origin were scored; headaches (yes; they feel like they come from the neck/jaw/neck and jaw/can't pinpoint the cause, no more than I believe is normal), grinding or clenching of the teeth, experienced pain/discomfort in the jaw, the existence of stiff or sore neck muscles, and fullness in the ears. The effect of conditions on the experienced tinnitus (e.g., psychological factors, movements, and activities) was scored in five categories, ranging from "made it a lot worse" to "made is a lot better."

\section{Statistical Analysis}

Descriptive analysis was used to assess the characteristics of participants and experienced tinnitus using means and SDs. Univariable logistic regression was used to calculate the relative odds of undergoing treatment or not and the effect of treatment by the different variables. Univariate linear regression was used to calculate the effect of physical conditions on awareness, loudness, and annoyance. All the data was analyzed using the R-statistics (www.R-project.org, R Core team 2013, Vienna, Austria) and the SPSS software (version 25, IBM Corp., Armonk, NY, USA). A statistically significant result will be defined as $p<0.05$.
TABLE 1 | Baseline characteristics of participants to surveys in numbers of participants (and \%).

\begin{tabular}{|c|c|c|}
\hline & $\begin{array}{c}\text { Survey causes and } \\
\text { treatment }(n=5,017) \\
\qquad N(\%)\end{array}$ & $\begin{array}{c}\text { Survey physical } \\
\text { links }(n=6,115) \\
N(\%)\end{array}$ \\
\hline Age (yrs) & & $54.1(13.8)^{*}$ \\
\hline$<18$ years & $52(1.0)$ & \\
\hline $18-44$ years & $1,520(30.2)$ & \\
\hline $45-64$ years & 2,555 (50.9) & \\
\hline$>65$ years & $874(17.4)$ & \\
\hline Prefer not to say & $16(0.3)$ & \\
\hline \multicolumn{3}{|l|}{ Gender } \\
\hline Male & 2,852 (56.8) & $3,154(51.6)$ \\
\hline Female & 2,132 (42.5) & 2,925 (47.8) \\
\hline Transgender & $17(0.3)$ & $17(0.3)$ \\
\hline Prefer not to say & $16(0.3)$ & $19(0.3)$ \\
\hline \multicolumn{3}{|l|}{ Onset of tinnitus } \\
\hline$<3$ months & $286(5.7)$ & \\
\hline 4-6 months & $257(5.1)$ & \\
\hline >6-12 months & $451(9.0)$ & \\
\hline$>1-5$ years & $1,777(35.4)$ & \\
\hline$>5-10$ years & $821(16.4)$ & \\
\hline >10-20 years & $688(13.7)$ & \\
\hline$>20$ years & $737(14.7)$ & \\
\hline Tinnitus duration (yrs) & & $4.4(9.2)^{\star}$ \\
\hline \multicolumn{3}{|l|}{ Primary cause } \\
\hline Don’t know & $1,440(28.7)$ & $1,618(27.6)$ \\
\hline Virus & $384(7.7)$ & $476(8.1)$ \\
\hline Ototoxic & $229(4.6)$ & $304(5.2)$ \\
\hline Acoustic trauma & $481(9.6)$ & $624(10.7)$ \\
\hline Age-related $\mathrm{HL}$ & $144(2.9)$ & $250(4.3)$ \\
\hline Otosclerosis & $30(0.6)$ & $48(0.8)$ \\
\hline Noise-induced HL & $689(13.7)$ & $748(12.8)$ \\
\hline Barotrauma & $33(0.7)$ & $65(1.1)$ \\
\hline Sudden HL & $120(2.4)$ & $237(4.0)$ \\
\hline Allergy & $20(0.4)$ & $28(0.4)$ \\
\hline Psychological & $243(4.8)$ & $269(0.5)$ \\
\hline TMJ & $74(1.5)$ & $141(2.4)$ \\
\hline Dental treatment & $34(0.7)$ & $51(0.09)$ \\
\hline Head or neck injury & $140(2.8)$ & $217(3.7)$ \\
\hline Meniere's & $116(2.3)$ & $170(2.9)$ \\
\hline Ear wax procedure & $50(1.0)$ & $69(1.1)$ \\
\hline Ear wax build up & $31(0.6)$ & $36(0.6)$ \\
\hline Metabolic & $19(0.4)$ & $55(0.9)$ \\
\hline Spontaneous onset & $322(6.4)$ & - \\
\hline Other & $418(8.3)$ & $340(5.8)$ \\
\hline Eustachian tube dysfunction & - & $108(1.8)$ \\
\hline Missing & - & 261 \\
\hline \multicolumn{3}{|l|}{ Hyperacusis } \\
\hline No & 1,893 (37.7) & \\
\hline Mildly & $1,301(25.9)$ & \\
\hline Moderately & 1,167 (23.3) & \\
\hline Severely & $456(9.1)$ & \\
\hline
\end{tabular}


TABLE 1 | Continued

\begin{tabular}{|c|c|c|}
\hline & $\begin{array}{c}\text { Survey causes and } \\
\text { treatment }(n=5,017) \\
\qquad N(\%)\end{array}$ & $\begin{array}{c}\text { Survey physical } \\
\text { links }(n=6,115) \\
\qquad N(\%)\end{array}$ \\
\hline Don’t know & $200(4.0)$ & \\
\hline \multicolumn{3}{|l|}{ Pulsatile tinnitus } \\
\hline Yes & $889(17.7)$ & $630(10.3)$ \\
\hline No & $3,651(72.8)$ & $5,485(89.7)$ \\
\hline \multicolumn{3}{|l|}{ Somatic tinnitus } \\
\hline Yes & $1,647(32.8)$ & \\
\hline No & $2,950(58.8)$ & \\
\hline \multicolumn{3}{|l|}{ Hearing loss } \\
\hline No & $1,837(36.6)$ & $1,219(19.9)$ \\
\hline Mild HL & 2,139 (42.6) & $2,071(33.9)$ \\
\hline Moderate HL & $742(14.8)$ & $1,380(22.6)$ \\
\hline Severe HL & $299(6.0)$ & $383(6.3)$ \\
\hline Not diagnosed, but think so & & $522(8.5)$ \\
\hline Not diagnosed, but don't think so & & $540(8.8)$ \\
\hline Tinnitus awareness ${ }^{\star}(0-10)$ & $8.6(2.2)$ & $75.4(27.6)^{\#}$ \\
\hline Tinnitus bothering ${ }^{\star}(0-10)$ & $6.7(3.0)$ & \\
\hline Tinnitus frustration* $(0-10)$ & $5.7(3.1)$ & \\
\hline Tinnitus loudness ${ }^{\star}(0-10)$ & & $6.9(2.2)$ \\
\hline Tinnitus annoyance* $(0-10)$ & & $6(3.0)$ \\
\hline \multicolumn{3}{|l|}{ Tinnitus caused } \\
\hline Stress & 2,479 (49.4) & \\
\hline Anxiety & 2,332 (46.5) & \\
\hline Panic-attacks & $758(15.1)$ & \\
\hline Depression & $1,697(33.8)$ & \\
\hline Concentration problems & $2,179(43.4)$ & \\
\hline OCD type condition & $189(3.8)$ & \\
\hline Insomnia & $1,955(39.0)$ & \\
\hline Alcohol abuse & $175(3.5)$ & \\
\hline Drug abuse & $72(1.4)$ & \\
\hline Other & $356(7.1)$ & \\
\hline \multicolumn{3}{|l|}{ Tinnitus pattern } \\
\hline No changes in volume & & $1,860(30.4)$ \\
\hline Grows louder during day & & $1,201(19.6)$ \\
\hline Gets quieter during day & & $351(5.7)$ \\
\hline Changes without pattern & & $2,703(44.2)$ \\
\hline \multicolumn{3}{|l|}{ Effect of sounds on tinnitus } \\
\hline Some make it worse & & $2,285(37.4)$ \\
\hline Some worse, some better & & $950(15.5)$ \\
\hline Some make it better & & $645(10.5)$ \\
\hline No difference & & 2,235 (36.5) \\
\hline \multicolumn{3}{|l|}{ Tinnitus louder by movement } \\
\hline Pressing jaw to the side & & $902(14.7)$ \\
\hline Pressing jaw backwards & & $858(14.0)$ \\
\hline Pressing jaw outwards & & $1,304(21.3)$ \\
\hline Pushing against forehead & & $1,090(17.8)$ \\
\hline Clenching teeth & & $1,419(23.2)$ \\
\hline Tilting head backwards & & $903(14.8)$ \\
\hline No change head /jaw movement & & $3,437(56.2)$ \\
\hline
\end{tabular}

When indicated with * mean (SD) are given.

\#Score on a scale 0-100 instead of 0-10.

$H L$, hearing loss.

\section{RESULTS}

\section{Survey "Treatment for Tinnitus" Characteristics of Cohort}

A total of 5,017 questionnaires were completed. The majority of participants were men $(n=2,852 ; 56.8 \%)$. A total of 2,555 (50.9 $\%)$ participants were in the age range of 45-64 years of age and $1,520(30.2 \%)$ were between 18 and 44 years of age. A total of 52 (1\%) participants were under the age of 18 years (and 16 preferred not to say their age; $0.3 \%$ ) (Table 1 ). In the majority of cases, the primary cause of tinnitus was scored as unknown $(n=1,440$; $28.7 \%)$. Noise-induced hearing loss $(n=689,13.7 \%)$ and acoustic trauma ( $n=481,9.6 \%)$ were scored as second and third most common causes. Most participants experienced some form of hyperacusis $(n=2,924,58.3 \%)$ and reported to have some degree of hearing loss ranging from mild to severe $(n=3,180,63.4 \%)$.

\section{Tinnitus Treatment and Effect}

Of the 5,017 participants in this survey, 2,914 (58.1\%) used one or more therapies (range 1-21) currently or in the past with a median of three treatments per participant. Of the 2,914 treated patients, $1,755(60.2 \%)$ reported the use of 1 3 different listed treatments, 771 (26.3\%) 4-6 treatments, and 388 (13.3\%) even $\geq 7$ treatments. Of those using therapy, $1,562(31.1 \%)$ used self-administered sound therapy, 1,157 (23.1\%) used supplements/herbal medicines, 785 (15.6\%) used antidepressants, 621 (12.4\%) used acupuncture, and 641 (12.8\%) used other treatments not listed. A total of 681 (13.8\%) participants reported using a hearing aid and $503(10.0 \%)$ used an in-ear masker (Table 2). Only 371 (7\%) participants used CBT, with a similar number using Tinnitus Retraining Therapy (TRT) $(n=370 ; 7.4 \%)$.

For the majority of therapies, most participants reported that there were no changes due to the therapy (Table 2). Only in case of hearing aids, self-administered sound therapy, and retigabine usage, the majority of participants noted a small improvement due to the treatment. Overall, only a small number of participants reported any degree of worsening caused by the experienced therapy, except for surgical treatments (treatment not specified). For the latter, in seven out of 36 cases (19.4\%), this was scored as "made it a lot worse."

\section{Comparison of Participants With and Without Therapy}

Table 3 shows the outcomes for the comparison of participants using tinnitus therapy compared to those not using it. Being female [odds ratio (OR) 0.83 (95\% CI 0.74-0.93, $p<0.01$ )], having mild [OR $1.43(95 \%$ CI $1.12-1.83, p<0.01)$ ] or moderate hearing loss [OR $1.40(96 \%$ CI $1.10-1.78, p<0.01)$ ], and moderate [OR 1.70 (95\% CI $1.26-2.30, p<0.01)$ ] or severe [OR 1.78 (95\% CI 1.32-2.41, $p<0.01$ )] hyperacusis were statistically significantly associated with higher odds of using (or having used) tinnitus treatment. Having higher tinnitus impact on daily life awareness [OR 1.05 (95\% CI 1.02-1.08, $p$ $<0.01$ )], bothersomeness [OR 1.08 (95\% CI 1.06-1.10, $p<$ $0.01)$ ] or frustration [OR 1.12 (96\% CI 1.10-1.14, $p<0.01)$ ], or experiencing physical and psychological effects by the tinnitus 
TABLE 2 | Numbers of participants which underwent a treatment out of survey "Causes and Treatment" $(n=5,017)$ with outcomes per treatment [numbers according to scored outcome per treatment and (\%)].

\begin{tabular}{|c|c|c|c|c|c|c|}
\hline Therapy type & $\begin{array}{l}\text { Participants which } \\
\text { used therapy } \\
N(\%)\end{array}$ & $\begin{array}{c}\text { Big improvement } \\
N(\%)\end{array}$ & $\begin{array}{c}\text { Small } \\
\text { improvement } \\
N(\%)\end{array}$ & No change $\boldsymbol{N}(\%)$ & $\begin{array}{c}\text { Made me slightly } \\
\text { worse } \\
N(\%)\end{array}$ & $\begin{array}{c}\text { Made me a lot } \\
\text { worse } \\
N(\%)\end{array}$ \\
\hline TRT & $370(7.4)$ & $50(13.5)$ & $134(36.2)$ & $164(44.3)$ & $16(4.3)$ & $6(1.6)$ \\
\hline In-ear masker & $503(10.0)$ & $34(6.8)$ & $199(39.6)$ & $227(45.1)$ & $32(6.4)$ & $11(2.2)$ \\
\hline CBT & $371(7.4)$ & $46(12.4)$ & $158(42.6)$ & $159(42.9)$ & $5(1.3)$ & $3(0.8)$ \\
\hline Psychiatrist & $298(5.9)$ & $18(6.0)$ & $89(29.9)$ & $174(58.4)$ & $8(2.7)$ & $9(3.0)$ \\
\hline Psychologist & $388(7.7)$ & $36(9.3)$ & $137(35.3)$ & $201(51.8)$ & $9(2.3)$ & $5(1.3)$ \\
\hline Neuromonics & $95(1.9)$ & $6(6.3)$ & $20(21.1)$ & $62(65.3)$ & $4(4.2)$ & $3(3.2)$ \\
\hline SoundCure & $144(2.9)$ & $8(5.6)$ & $43(29.9)$ & $80(55.6)$ & $7(4.9)$ & $6(4.2)$ \\
\hline Acoustic neuromodulation & $120(2.4)$ & $7(5.8)$ & $37(30.8)$ & $73(60.8)$ & $2(1.7)$ & $1(0.8)$ \\
\hline Notched music therapy & $223(4.4)$ & $4(1.8)$ & $64(28.7)$ & $145(65.0)$ & $10(4.5)$ & $0(0.0)$ \\
\hline Hearing aid & $681(13.6)$ & $98(14.4)$ & $276(40.5)$ & $236(34.7)$ & $44(6.5)$ & $27(4.0)$ \\
\hline Self-administered sound therapy & $1,562(31.1)$ & $146(9.3)$ & $798(51.1)$ & $584(37.4)$ & $26(1.7)$ & $8(0.5)$ \\
\hline Bio or neuro feedback / meditation & $270(5.4)$ & $21(7.8)$ & $112(41.5)$ & $131(48.5)$ & $5(1.9)$ & $1(0.4)$ \\
\hline Antidepressants & $785(15.6)$ & $64(8.2)$ & $280(35.7)$ & $340(43.3)$ & $68(8.7)$ & $33(4.2)$ \\
\hline GABA type drugs & $237(4.7)$ & $23(9.7)$ & 86 (36.3) & $104(43.9)$ & $16(6.8)$ & $8(3.4)$ \\
\hline Retigabine & $53(1.1)$ & $15(28.3)$ & $18(34.0)$ & $16(30.2)$ & $2(3.8)$ & $2(3.8)$ \\
\hline Transcranial stimulation & $45(0.9)$ & $2(4.4)$ & $8(17.8)$ & $31(68.9)$ & $2(4.4)$ & $2(4.4)$ \\
\hline НВOT & $46(0.9)$ & $2(4.3)$ & $11(23.9)$ & $32(69.6)$ & $1(2.2)$ & $0(0.0)$ \\
\hline Steroids & $346(6.9)$ & $23(6.6)$ & $82(23.7)$ & $218(63.0)$ & $11(3.2)$ & $12(3.5)$ \\
\hline Low-level laser treatment & $65(1.3)$ & $4(6.2)$ & $11(16.9)$ & $42(64.6)$ & $7(10.8)$ & $1(1.5)$ \\
\hline Off-label medication & $312(6.2)$ & $9(2.9)$ & $76(27.2)$ & $217(69.6)$ & $7(2.2)$ & $3(1.0)$ \\
\hline Surgical procedure & $36(0.7)$ & $1(2.8)$ & $8(22.2)$ & $17(47.2)$ & $3(8.3)$ & 7 (19.4) \\
\hline Acupuncture & $621(12.4)$ & $13(2.1)$ & $148(23.8)$ & $445(71.7)$ & $12(1.9)$ & $3(0.5)$ \\
\hline Chiropractor & $489(9.7)$ & $11(2.2)$ & $97(19.8)$ & $367(75.1)$ & $10(2.0)$ & $4(0.8)$ \\
\hline Supplements / herbal medicines & $1,157(23.1)$ & $32(2.8)$ & 247 (21.3) & $860(74.3)$ & $12(1.0)$ & $6(0.5)$ \\
\hline Tinnitus cure eBooks & $254(5.1)$ & $8(3.1)$ & $53(20.9)$ & $189(74.4)$ & $2(0.8)$ & $2(0.8)$ \\
\hline Homeopathic treatment & $425(8.5)$ & $13(3.1)$ & $79(18.6)$ & $323(76.0)$ & $6(1.4)$ & $4(0.9)$ \\
\hline Other treatment & $641(12.8)$ & $130(20.3)$ & $224(34.9)$ & $261(40.7)$ & $14(2.2)$ & $12(1.9)$ \\
\hline
\end{tabular}

were all significantly statistically associated with higher odds of having tinnitus treatment (Table 3).

\section{Survey Physical Links Characteristics of Cohort}

In total, 6,115 participants completed the survey "Physical Links" (Table 1). The majority of participants were men $[n=3,154$ (51.6\%)]. Mean age of the participants was 54.1 years (SD 13.8). Similar to survey one, the most reported primary cause of tinnitus was unknown ( $n=1,618,27.6 \%)$, noise induced hearing loss $(n=$ $748,12.8 \%)$, and acoustic trauma $(n=624,10.7 \%)$. The majority of cases had some (diagnosed or self-reported) degree of hearing loss $(n=4,356,71.2 \%)$.

\section{Physical and Psychological Factors of Influence on the Experienced Tinnitus}

The largest percentage of responders indicated that sleep (good sleep, bad sleep, or napping) made no difference in tinnitus experience [respectively, in 2,304 (37.7\%), 1,875 (30.7\%), and 2,668 (43.6\%) of cases] (Table 4). Physical exercise made no difference in tinnitus experience for the majority of participants [intense work-out $(2,078,34.0 \%)$, moderate $(2,496,40.8 \%)$, or light exercise $(3,510,57.4 \%)]$. Both anxiety and stress were mostly scored as making tinnitus a little worse [respectively, in 2,094 $(34.2 \%)$ and $2,167(35.4 \%)$ of cases] or a lot worse [respectively, in $1,662(27.2 \%)$ and $1,809(29.6 \%)$ ]. Specific movements of the jaw, head, and teeth made the tinnitus louder in 14.0-23.2\% of cases depending on the type, whereby about half of the respondents experienced no change to their tinnitus caused by movement of the head or jaw $(n=3,437,56.2 \%)$ (Table 1).

\section{Characteristics Related to the Experienced Tinnitus}

Table 5 shows the outcomes for the univariate regression analysis for the effect of demographics, etiology, and physical factors and comorbidities on the experienced tinnitus during the past week. Statistically significant outcomes are described. Women experienced a statistically significant higher tinnitus awareness ( $\beta$ 5.36, 95\% CI 3.98-6.75, $p<0.01$ ), annoyance $(\beta 0.54,95 \%$ CI $0.39-0.68$; $p<0.01$ ), and loudness $(\beta 0.38$, 95\% CI $0.27-$ $0.49, p<0.01)$ compared to men. Overall, sudden hearing loss, head or neck injury, Meniere's disease, headaches, fullness in the ears, and all categories of hearing loss were significantly positively 
TABLE 3 | Outcomes of univariate regression analysis of tinnitus impact and characteristics and psychological/ physical effects of tinnitus between those with and without having treatment (survey "Treatment for tinnitus").

\begin{tabular}{|c|c|c|c|}
\hline \multicolumn{4}{|c|}{ Comparison of participants with and without therapy } \\
\hline & Treatment & $\begin{array}{c}\text { No } \\
\text { treatment }\end{array}$ & OR \\
\hline & $(n=2,914)$ & $(n=2,103)$ & (95\% Cl, $p$-value) \\
\hline & $N(\%)$ & $N(\%)$ & \\
\hline \multicolumn{4}{|l|}{ Gender } \\
\hline Male & $1,712(58.8)$ & $1,140(54.2)$ & Ref \\
\hline Female & $1,181(40.5)$ & $951(45.2)$ & $0.83(0.74-0.93, p<0.01)$ \\
\hline Transgender & $11(0.4)$ & $6(0.3)$ & $1.22(0.46-3.55, p=0.70)$ \\
\hline Prefer not to say & $10(0.3)$ & $6(0.3)$ & $1.11(0.41-3.27, p=0.84)$ \\
\hline \multicolumn{4}{|l|}{ Hearing loss } \\
\hline None & 1,097 (37.6) & $740(32.5)$ & Ref \\
\hline Mild & $1,265(43.4)$ & $874(41.6)$ & $1.43(1.12-1.83, p<0.01)$ \\
\hline Moderate & $400(13.7)$ & $342(16.3)$ & $1.40(1.10-1.78, p<0.01)$ \\
\hline Severe & $152(5.2)$ & $147(7.0)$ & $1.13(0.87-1.48, p=0.37)$ \\
\hline \multicolumn{4}{|l|}{ Hyperacusis } \\
\hline No & $1,006(34.5)$ & $887(42.2)$ & Ref \\
\hline Mild & 795 (27.3) & $506(24.1)$ & $1.23(0.92-1.65, p=0.10)$ \\
\hline Moderate & $726(24.9)$ & $441(21.0)$ & $1.70(1.26-2.30, p<0.01)$ \\
\hline Severe & $291(10.0)$ & $165(7.8)$ & $1.78(1.32-2.41, p<0.01)$ \\
\hline Don’t know & $96(3.3)$ & $104(4.9)$ & $1.91(1.36-2.68, p<0.01)$ \\
\hline Tinnitus awareness & $8.7(2.1)$ & $8.5(2.3)$ & $1.05(1.02-1.08, p<0.01)$ \\
\hline Tinnitus bothering & $6.9(2.9)$ & $6.3(3.0)$ & $1.08(1.06-1.10, p<0.01)$ \\
\hline Tinnitus frustration & $6.1(3.1)$ & $5.0(3.1)$ & $1.12(1.10-1.14, p<0.01)$ \\
\hline \multicolumn{4}{|l|}{ Pulsatile tinnitus } \\
\hline No & $488(16.7)$ & $401(19.1)$ & Ref \\
\hline Yes & 2,168 (74.4) & 1,483 (70.5) & $1.03(0.83-1.30, p=0.78)$ \\
\hline Unsure & $258(8.9)$ & $219(10.4)$ & $1.24(1.02-1.50, p=0.03)$ \\
\hline \multicolumn{4}{|l|}{ Somatic tinnitus } \\
\hline No & $1,056(36.2)$ & $591(28.1)$ & Ref \\
\hline Yes & $1,643(56.4)$ & $1,307(62.1)$ & $1.70(1.37-2.12, p<0.01)$ \\
\hline Unsure & $215(7.4)$ & $205(9.7)$ & $1.20(0.98-1.47, p=0.08)$ \\
\hline \multicolumn{4}{|l|}{$\begin{array}{l}\text { Tinnitus caused } \\
\text { me.... }\end{array}$} \\
\hline Stress & & & $3.09(2.75-3.47, p<0.01)$ \\
\hline Yes & $1,140(55.9)$ & $1,398(55.1)$ & \\
\hline No & $1,774(71.6)$ & $705(28.4)$ & \\
\hline Anxiety & & & $2.94(2.62-3.31, p<0.01)$ \\
\hline Yes & $1,242(46.3)$ & $1,443(53.7)$ & \\
\hline No & $1,672(71.7)$ & $660(28.3)$ & \\
\hline Panic attacks & & & $2.85(2.38-3.42, p<0.01)$ \\
\hline Yes & $2,327(54.6)$ & $1,932(45.4)$ & \\
\hline No & $587(77.4)$ & $171(22.6)$ & \\
\hline Depression & & & $3.31(2.91-3.77, p<0.01)$ \\
\hline Yes & $1,624(48.9)$ & $1,696(51.1)$ & \\
\hline No & 1,290 (76.0) & $407(24.0)$ & \\
\hline $\begin{array}{l}\text { OCD type } \\
\text { condition }\end{array}$ & & & $1.79(1.31-2.49, p<0.01)$ \\
\hline Yes & $2,780(57.6)$ & $2,048(42.4)$ & \\
\hline No & $134(70.9)$ & $55(29.1)$ & \\
\hline
\end{tabular}

(Continued)
TABLE 3 | Continued

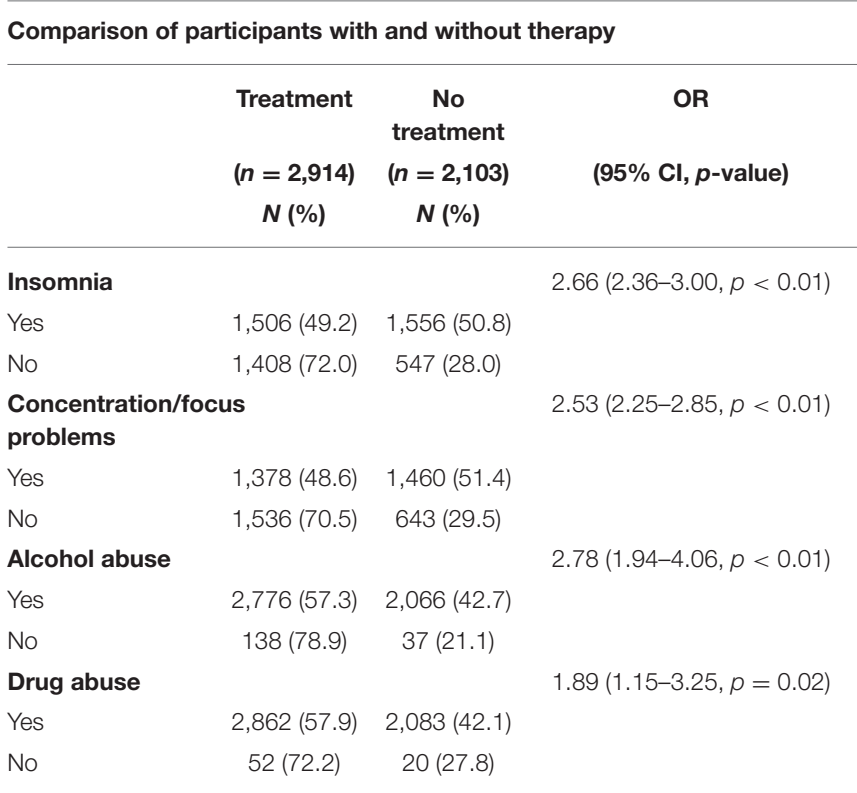

Ref, reference category.

associated with the experienced tinnitus loudness, annoyance, and awareness. Other factors showed statistically inconsistent relations compared to the categories of tinnitus impact.

\section{DISCUSSION}

Most of the current knowledge about people with tinnitus and treatment outcomes is retrieved from studies examining a sample of patients visiting tinnitus health care centers for diagnosis, therapy, or counseling. Considering the lack of curative treatments, it is not surprising that people with tinnitus keep looking for symptom relief with the help of complementary or alternative medicine (14). By assessing the characteristics and effect of therapy of people with tinnitus responding to a worldwide internet-based survey, we were able to gain insight in a group of people with tinnitus not necessarily visiting a health care center for their tinnitus. Thereby, we have to keep in mind that these results do reflect thoughts and assumptions of participants about the cause of tinnitus, tinnitus-related difficulties, and experienced treatment effects.

Half of our patients used more than one therapy. Of those using treatment, about 2 out of 5 people used $>4$ tinnitus therapies, including conventional and non-conventional tinnitus therapies. This number might not be representative for the average tinnitus patient, but do reflect a self-selecting group of people who struggle with their tinnitus enough to seek help online and willing to respond to a survey posted on Tinnitus Talk. Thereby, someone who is not bothered at all by their tinnitus is unlikely to seek out online support groups and would not notice the survey requests. Besides this, it is possible that patients with more help-seeking behavior are more prone to fill out the online questionnaires. Still, this outcome is of highly interest as it reflects 
TABLE 4 | Effect of physical and psychological factors on experienced tinnitus out of survey physical links $(n=6,115)$.

\begin{tabular}{|c|c|c|c|c|c|c|c|}
\hline \multicolumn{2}{|c|}{ “.....made the tinnitus..” } & \multirow{2}{*}{$\begin{array}{c}\text { A lot worse } \\
\qquad \begin{array}{c}\boldsymbol{N}(\%) \\
303(5)\end{array}\end{array}$} & \multirow{2}{*}{$\begin{array}{c}\text { A little worse } \\
\qquad \boldsymbol{N}(\%) \\
469(7.7)\end{array}$} & \multirow{2}{*}{$\begin{array}{c}\text { No difference } \\
\qquad \boldsymbol{N}(\%)\end{array}$} & \multirow{2}{*}{$\begin{array}{c}\text { A little better } \\
\text { N (\%) }\end{array}$} & \multirow{2}{*}{$\begin{array}{c}\text { A lot better } \\
\text { N (\%) } \\
710(11.6)\end{array}$} & \multirow{2}{*}{$\begin{array}{c}\text { Unsure/don't know } \\
\qquad \boldsymbol{N}(\%) \\
858(14.0)\end{array}$} \\
\hline Sleep & Good sleep & & & & & & \\
\hline & Bad sleep & $1,406(23.0)$ & 1,951 (31.9) & $1,875(30.7)$ & $53(0.9)$ & $17(0.3)$ & $813(13.3)$ \\
\hline & Napping & $572(9.4)$ & $832(13.6)$ & 2,668 (43.6) & $453(7.4)$ & $54(0.9)$ & $1,536(25.1)$ \\
\hline \multirow[t]{3}{*}{ Exercise } & Intense workout & $351(5.7)$ & $844(13.8)$ & $2,078(34.0)$ & $385(6.3)$ & $111(1.8)$ & 2,346 (38.4) \\
\hline & Moderate exercise & $223(3.6)$ & $901(14.7)$ & 2,496 (40.8) & $588(9.6)$ & $116(1.9)$ & 1,791 (29.3) \\
\hline & Light exercise & $80(1.3)$ & $494(8.1)$ & $3,510(57.4)$ & $764(12.5)$ & $138(2.3)$ & $1,129(18.5)$ \\
\hline \multirow[t]{2}{*}{ Psychological } & Anxiety & 1,662 (27.2) & 2,094 (34.2) & $1,294(21.2)$ & $23(0.4)$ & $10(0.2)$ & 1,032 (16.9) \\
\hline & Stress & 1,809 (29.6) & $2,167(35.4)$ & $1,186(19.4)$ & $21(0.30)$ & $6(0.10)$ & $926(15.1)$ \\
\hline
\end{tabular}

Frequencies and (\%).

the ongoing search for tinnitus relief in people bothered by their tinnitus. These people who are struggling are the ones who need help and better treatments, thus exactly the subgroup of interest for researchers and clinicians for future improvements of tinnitus health care.

Being female, having higher tinnitus impact on daily life, and experiencing physical or psychological effects by the tinnitus were all associated with statistically significant higher odds of using tinnitus treatment. Self-administered sound therapy, supplements/herbal medicines, antidepressants, and acupuncture were most commonly used. This can be related to the costs or accessibility of treatments. Most (sub)types of listed sound therapies, psychological therapies, as well as drug therapies (such as antidepressants, GABA-type drugs, and retigabine) were rated as having a positive effect on their tinnitus by the reports of the participants. This is of interest considering the lack of positive effects to tinnitus found in systematic reviews so far, assessing most of these therapies applied in clinical studies, or the lack of evidence for others $(10,11,15)$. Currently, by its proven effectiveness, cognitive behavior therapy is advocated to treat tinnitus-related distress in those whose tinnitus is causing an impact on their well-being and activities $(10,16)$. In our study, $55 \%$ of people who tried CBT experienced a degree of improvement, where about $40 \%$ reported to have no change to their tinnitus. Although, to explain the positive results on many therapies reported by participants, we need to keep in mind that the expericend effect can be influenced by a placebo effect as described for several tinnitus therapies (17). Besides this, many participants have undergone multiple treatments by which it can be difficult to recall and assess the effect of a specific (simultaneous or sequential) therapy (many years) afterwards. On the other hand, the order effect (the order in which the participant received a therapy may affect the outcome), measurement (or "judgement") errors of repeated individual evaluations, and the classification of therapy outcomes into categories of effect could distort the real effect of therapies. Lastly, out of this survey, we were not able to verify if tinnitus treatments were used by the stated indication criteria or were provided according to treatment standards (such as for psychological treatments), which could influence the experienced effectiveness. To sort these effects out, influencing outcomes of (multiple) therapies, prospective, protocollized, and, when possible, randomized controlled studies would be needed. Considering the possible placebo effect of tinnitus therapies, it would be beneficial to describe these effects in more detail.

Anxiety and stress, as well as being female, having a higher age, certain listed causes, experiencing headaches, fullness in the ears, and any degree of hearing loss were statistically significant associated with a higher experienced tinnitus loudness, annoyance, or awareness. However, the latter effect sizes were mostly $<1$ point difference on loudness and annoyance scores (both on a scale from 0 to 10 ) or $<10$ points change on awareness scores (on a scale from 0 to 100) and therefore not deemed to be clinically relevant. From the few previous reports examining the relationship between the impact of tinnitus and tinnitus (un)related characteristics, the study from Hiller and Goebel is worthwhile to mention. In a similar nonclinical sample of members of the German Tinnitus League, they demonstrated that tinnitus loudness and annoyance were higher in subjects with hearing loss, vertigo, and hyperacusis (18). Secondly, substantially higher rates of loudness and annoyance were found in those whose tinnitus was due to conductive hearing loss, severe head injury, or neurologic disease (18). But in this study, the degrees of tinnitus loudness and annoyance were categorized in grades of severity, hindering comparison of effect-sizes with outcomes of our study.

The strength of this study is the worldwide cohort, in which participants were not necessarily related to a specific health care center or clinic. Nonetheless, several limitations need to be addressed. First of all, recall bias could have been introduced by the fact that in both surveys, people were asked to answer questions about their previous experiences with factors of influence on their tinnitus and the effect of tinnitus treatments. Secondly, outcomes of the study rely on data of two surveys which could be completed by the same participants. Thirdly, the survey was written in English language which could have introduced limitations for people to attend the survey and limits the generalizability of outcomes. Fourthly, the presence or absence of symptoms or diseases was scored according to the subjective judgement of the participant by which outcomes could deviate from objective measures such as for hearing loss. Next, survey questions on diseases and symptoms such as the 
TABLE 5 | Outcome of univariate regression analysis of effect of demographics, etiology, and physical factors/comorbidities on experienced impact of tinnitus during the past week (survey physical links).

\begin{tabular}{|c|c|c|c|c|c|c|c|c|c|c|}
\hline & \multirow[b]{2}{*}{$N$} & \multicolumn{3}{|c|}{ Tinnitus loudness } & \multicolumn{3}{|c|}{ Tinnitus annoyance } & \multicolumn{3}{|c|}{ Tinnitus awareness } \\
\hline & & Mean (SD) & B (95\%Cl) & $P$ & Mean (SD) & B (95\% Cl) & $P$ & Mean (SD) & B (95\%Cl) & $P$ \\
\hline Age & & $7.0(2.2)$ & 0.03 (0.02-0.03) & $<0.01$ & $6.9(3.0)$ & $0.02(0.01-0.02)$ & $<0.01$ & $68.5(27.6)$ & $0.23(0.27-0.36)$ & $<0.01$ \\
\hline \multicolumn{11}{|l|}{ Sex } \\
\hline Male & 3,139 & $6.8(2.2)$ & Ref & - & $6.6(2.9)$ & Ref & - & $66.9(28.2)$ & Ref & - \\
\hline Female & 2,918 & $7.2(2.3)$ & $0.38(0.27-0.49)$ & $<0.01$ & $7.1(3.0)$ & $0.54(0.39-0.68)$ & $<0.01$ & $71.3(26.8)$ & $5.36(3.98-6.75)$ & $<0.01$ \\
\hline Transgender & 17 & $7.3(2.6)$ & $0.52(-0.53-1.57)$ & 0.33 & $7.9(2.9)$ & $1.33(-0.08-2.74)$ & 0.06 & $75.1(32.1)$ & $9.18(-3.94-22.30)$ & 0.17 \\
\hline Prefer not to say & 19 & $7.8(2.1)$ & $1.01(0.02-2.01)$ & 0.05 & $7.3(3.3)$ & $0.65(-0.68-1.98)$ & 0.34 & $71.6(27.6)$ & $5.65(-6.77-18.06)$ & 0.37 \\
\hline \multicolumn{11}{|l|}{ Etiology } \\
\hline Unknown & 1,618 & $6.9(2.1)$ & Ref & - & $6.8(3.0)$ & Ref & - & $68.13(28.24)$ & Ref & - \\
\hline Sudden HL & 237 & $7.5(2.2)$ & $0.58(0.28-0.89)$ & $<0.01$ & $7.6(3.1)$ & $0.84(0.44-1.24)$ & $<0.01$ & $74.65(27.83)$ & $6.52(2.76-10.27)$ & 0.01 \\
\hline Noise induced HL & 748 & $7.0(2.1)$ & $0.14(-0.5-0.33)$ & 0.16 & $6.7(2.9)$ & $-0.04(-0.30-0.21)$ & 0.75 & $66.56(27.50)$ & $-1.57(-3.96-0.81)$ & 0.20 \\
\hline Noise trauma & 624 & $6.8(2.2)$ & $-0.7(-0.27-0.13)$ & 0.50 & $6.8(2.8)$ & $0.04(-0.24-0.31)$ & 0.79 & $65.25(27.70)$ & $-2.87(-5.42--0.33)$ & 0.03 \\
\hline Virus & 476 & $6.6(2.2)$ & $-0.27(-0.50--0.05)$ & 0.02 & $6.4(2.9)$ & $-0.37(-0.67--0.07)$ & 0.02 & $65.78(27.49)$ & $-2.35(-5.16-0.47)$ & 0.10 \\
\hline Ototoxic & 304 & $6.9(2.2)$ & $0.03(-0.24-0.30)$ & 0.84 & $7.0(2.8)$ & $0.28(-0.08-0.64)$ & 0.13 & $69.48(27.30)$ & $1.35(-2.3-4.72)$ & 0.43 \\
\hline Age related $\mathrm{HL}$ & 250 & $6.5(2.0)$ & $-0.36(-0.62--0.07)$ & 0.02 & $6.2(2.9)$ & $-0.53(-0.93--0.14)$ & 0.01 & $65.64(28.13)$ & $-2.49(-6.16-1.18)$ & 1.87 \\
\hline Eustachian tube dysfunction & 108 & $6.9(2.3)$ & $-0.02(-0.45-0.41)$ & 0.94 & $7.4(3.1)$ & $0.62(0.05-1.19)$ & 0.03 & $71.80(26.83)$ & $3.67(-1.70-9.03)$ & 0.18 \\
\hline Otosclerosis & 48 & $7.3(2.2)$ & $0.43(-0.21-1.06)$ & 0.19 & $6.8(3.0)$ & $0.02(-0.82-0.87)$ & 0.96 & $74.56(25.68)$ & $6.43(-1.47-14.34)$ & 0.11 \\
\hline Barotrauma & 65 & $7.3(2.4)$ & $0.36(-0.19-0.90)$ & 0.20 & $6.9(2.8)$ & $0.12(-0.61-0.85)$ & 0.74 & $69.86(28.23)$ & $1.73(-5.09-8.56)$ & 0.62 \\
\hline Allergy & 28 & $6.5(2.1)$ & $-0.43(-1.26-0.41)$ & 0.32 & $6.6(2.8)$ & $-0.16(-1.26-0.94)$ & 0.78 & $66.00(27.64)$ & $-2.13(-12.41-8.16)$ & 0.69 \\
\hline Psychosocial & 269 & $6.8(2.4)$ & $-0.15(-0.43-0.14)$ & 0.31 & $6.9(3.2)$ & $0.12(-0.26-0.51)$ & 0.52 & $66.29(28.57)$ & $-1.84(-5.39-1.72)$ & 0.31 \\
\hline TMJ & 141 & $6.5(2.2)$ & $-0.41(-0.79--0.03)$ & 0.04 & $6.9(2.8)$ & $0.10(-0.41-0.61)$ & 0.69 & $66.58(26.77)$ & $-1.55(-6.29-3.19)$ & 0.52 \\
\hline Dental treatment & 51 & $7.0(2.2)$ & $0.07(-0.54-0.69)$ & 0.81 & $6.8(3.1)$ & $0.02(-0.80-0.84)$ & 0.97 & $66.86(26.86)$ & $-1.27(-8.94-6.41)$ & 0.75 \\
\hline Head or neck injury & 217 & $7.6(2.4)$ & $0.71(0.40-1.03)$ & $<0.01$ & $7.9(2.8)$ & $1.12(0.70-1.53)$ & $<0.01$ & 74.66 (24.93) & $6.54(2.63-10.44)$ & $<0.01$ \\
\hline Meniere's & 170 & $7.5(2.4)$ & $0.58(0.23-0.92)$ & $<0.01$ & $7.3(2.9)$ & $0.51(0.04-0.97)$ & 0.03 & $74.08(27.04)$ & $5.95(1.60-10.30)$ & 0.03 \\
\hline Earwax procedure & 69 & $7.1(2.3)$ & $0.18(-0.35-0.72)$ & 0.50 & $7.7(2.8)$ & $0.91(0.20-1.62)$ & 0.01 & $75.59(24.37)$ & $7.47(0.83-14.10)$ & 0.03 \\
\hline Earwax build up & 36 & $5.8(2.3)$ & $-1.10(-1.83--0.37)$ & 0.01 & $6.6(2.9)$ & $-0.21(-1.18-0.76)$ & 0.67 & $58.58(29.26)$ & $-9.55(-18.64--0.45)$ & 0.04 \\
\hline Metabolic & 55 & $6.9(2.0)$ & $0.04(-0.55-0.63)$ & 0.90 & $6.4(2.8)$ & $-0.39(-1.18-0.41)$ & 0.34 & $65.95(22.69)$ & $-2.18(-9.58-5.21)$ & 0.56 \\
\hline Not listed & 340 & $7.3(2.3)$ & $0.40(0.14-0.66)$ & $<0.01$ & $7.2(3.1)$ & $0.43(0.09-0.77)$ & 0.02 & $74.41(26.14)$ & $6.28(3.06-9.50)$ & $<0.01$ \\
\hline Stiff/sore neck muscles & & & $-0.11(-0.36-0.14)$ & 0.38 & & $-0.15(-0.48-0.18)$ & $<0.01$ & & $-2.17(-5.25-0.91)$ & 0.17 \\
\hline No & 3,268 & $7.1(2.2)$ & & & $7.0(2.9)$ & & & $70.6(25.8)$ & & \\
\hline Yes & 5,787 & $7.0(2.2)$ & & & $6.9(3.0)$ & & & $68.4(27.7)$ & & \\
\hline Headaches & & & $0.43(0.31-0.54)$ & $<0.01$ & & $0.83(0.68-0.98)$ & $<0.01$ & & $6.15(4.73-7.57)$ & $<0.01$ \\
\hline No & 3,790 & $6.8(2.2)$ & & & $6.6(2.9)$ & & & $66.2(28.2)$ & & \\
\hline Yes & 2,325 & $7.2(2.2)$ & & & $7.4(2.9)$ & & & $72.4(26.3)$ & & \\
\hline Fullness in the ears & & & $0.37(0.25-0.48)$ & $<0.01$ & & $0.69(0.54-0.84)$ & $<0.01$ & & 5.89 (4.48-7.29) & $<0.01$ \\
\hline No & 2,460 & $6.7(2.2)$ & & & $6.5(3.0)$ & & & $65.0(28.6)$ & & \\
\hline Yes & 3,655 & $7.1(2.2)$ & & & $7.2(2.9)$ & & & $70.9(26.7)$ & & \\
\hline Pain/discomfort jaw & & & $0.19(-0.04-0.20)$ & 0.18 & & $0.34(0.18-0.49)$ & $<0.01$ & & $1.91(0.46-3.36)$ & 0.01 \\
\hline No & 3,949 & $6.9(2.2)$ & & & $6.8(3.0)$ & & & $67.9(28.0)$ & & \\
\hline Yes & 2,166 & $7.0(2.2)$ & & & $7.1(2.9)$ & & & $69.8(26.9)$ & & \\
\hline \multicolumn{11}{|l|}{ Hearing loss } \\
\hline No & 1,219 & $6.4(2.3)$ & Ref & - & $6.5(3.0)$ & Ref & - & $65.3(28.2)$ & Ref & - \\
\hline Mild HL & 2,071 & $6.9(2.2)$ & $0.46(0.31-0.62)$ & $<0.01$ & $6.8(2.9)$ & $0.30(0.09-0.51)$ & $<0.01$ & $67.7(27.3)$ & $2.43(0.50-4.35)$ & 0.01 \\
\hline Moderate HL & 3,803 & $7.5(2.1)$ & $1.08(0.92-1.25)$ & $<0.01$ & $7.3(2.9)$ & $0.74(0.52-0.97)$ & $<0.01$ & $73.6(25.7)$ & 8.29 (6.20-10.39) & $<0.01$ \\
\hline Severe HL & 83 & $8.2(2.3)$ & $1.71(1.47-1.96)$ & $<0.01$ & $8.1(2.9)$ & $1.57(1.23-1.90)$ & $<0.01$ & $80.8(26.0)$ & $15.53(12.40-18.65)$ & $<0.01$ \\
\hline Not diagnosed, but think so & 522 & $6.7(2.1)$ & $0.29(0.07-0.51)$ & 0.01 & $6.6(2.9)$ & $0.09(-0.21-0.40)$ & 0.53 & $66.5(27.7)$ & $1.26(-1.53-4.04)$ & 0.38 \\
\hline $\begin{array}{l}\text { Not diagnosed, but don't } \\
\text { think so }\end{array}$ & 540 & $6.2(2.2)$ & $-0.23(-0.44-0.01)$ & 0.04 & $6.1(3.0)$ & $-0.40(-0.69--0.10)$ & $<0.01$ & $59.7(28.8)$ & $-5.58(-8.34--2.82)$ & $<0.01$ \\
\hline
\end{tabular}

Ref, reference category; HL, hearing Loss.

In bold outcomes with a statistically significant result defined as a p-value $<0.05$. 
question phrased to assess hyperacusis (classically defined as a negative reactions to a sound only depending on its physical characteristics) could also encompass other subdivisions of decreased sound tolerance such as misophonia (in the literature defined as an abnormally strong reaction to a sound with a specific pattern and meaning to a given subject) (19). Therefore, outcomes need to be interpreted carefully and in line with the phrased question.

Regrettably, an effective treatment for tinnitus is still not available. As demonstrated in our study, more than $50 \%$ of participants used $>1$ type of conventional or nonconventional therapies, in many cases without proven (and experienced) benefit. From this outcome, one could question the accessibility of up-to-date information about the effectiveness of tinnitus treatments in current tinnitus health care for patients and health care providers. Optimization of this information would necessitate combined efforts of patients, physicians, and stakeholders and could potentially reduce the consumption of (non)conventional health care for tinnitus. On the other hand, the outcomes of our study created insights about experiences, thoughts, and beliefs of people with tinnitus, which could help in optimizing tinnitus health care of those in need for help. Moreover, the high number of individuals experiencing no clinical impact of treatment on tinnitus underlines the need for finding a personalized and accessible treatment for patients with tinnitus. Future research should investigate how physical, mental health, and tinnitus-related factors influence and predict the impact of tinnitus on peoples' lives and which factors explain the experienced effect of treatment on individuals. This could result in finding "subtypes" of patients with tinnitus that respond to

\section{REFERENCES}

1. van den Berge MJC, Free RH, Arnold R, de Kleine E, Hofman R, van Dijk JMC, et al. Cluster analysis to identify possible subgroups in tinnitus patients. Front Neurol. (2017) 8:1-7. doi: 10.3389/fneur.2017.00115

2. Moller AR, Salvi R, De Ridder D, Kleinjung T, Vanneste S. Pathology of tinnitus and hyperacusis-clinical implications. Biomed Res Int. (2015) 2015:24. doi: $10.1155 / 2015 / 608437$

3. McFerran DJ, Stockdale D, Holme R, Large CH, Baguley DM. Why is there no cure for tinnitus? Front Neurosci. (2019) 13:1-13. doi: 10.3389/fnins.2019.00802

4. Hoare DJ, Kowalkowski VL, Kang S, Hall DA. Systematic review and metaanalyses of randomized controlled trials examining tinnitus management. Laryngoscope. (2011) 121:1555-64. doi: 10.1002/lary.21825

5. Mertens G, De Bodt M, Van de Heyning P. Cochlear implantation as a long-term treatment for ipsilateral incapacitating tinnitus in subjects with unilateral hearing loss up to 10 years. Hear Res. (2016) 331:1-6. doi: 10.1016/j.heares.2015.09.016

6. Dobie RA. A review of randomized clinical trials in tinnitus. Laryngoscope. (1999) 109:1202-11. doi: 10.1097/00005537-199908000-00004

7. Hoare DJ, Edmondson-Jones M, Sereda M, Akeroyd MA, Hall D. Amplification with hearing aids for patients with tinnitus and coexisting hearing loss. Cochrane Database Syst Rev. (2014) 2014:CD010151. doi: 10.1002/14651858.CD010151.pub2

8. Martinez-Devesa P, Perera R, Theodoulou M, Waddell A. Cognitive behavioural therapy for tinnitus. Cochr Database Syst Rev. (2010) 2010:CD005233. doi: 10.1002/14651858.CD005233.pub3

9. Makar SK, Mukundan G, Gore G. Treatment of tinnitus: a scoping review. Int Tinnitus J. (2017). doi: 10.5935/0946-5448.20170027 certain types of therapy. Collaboration between research groups to combine data sets is the first step to achieve this goal.

\section{DATA AVAILABILITY STATEMENT}

The raw data supporting the conclusions of this article will be made available by the authors, without undue reservation.

\section{ETHICS STATEMENT}

The studies involving human participants were reviewed and approved by Ethical Committee of the University Medical Center Utrecht, Netherlands (local number: 19-134/C). The patients/participants provided their written informed consent to participate in this study.

\section{AUTHOR CONTRIBUTIONS}

AS wrote the manuscript text and interpreted the data. MV designed and conducted the surveys and revised the manuscript. $\mathrm{HG}$ revised the manuscript and assisted in drafting conclusion. JE and CW assisted in organizing the outcomes of the surveys. IS conducted the statistical analysis and assisted in writing the results. All authors reviewed the manuscript.

\section{SUPPLEMENTARY MATERIAL}

The Supplementary Material for this article can be found online at: https://www.frontiersin.org/articles/10.3389/fneur. 2021.778450/full\#supplementary-material

10. Lewis S, Chowdhury E, Stockdale D, Kennedy V. Assessment and management of tinnitus: Summary of NICE guidance. BMJ. (2020) 368:1-5. doi: 10.1136/bmj.m976

11. Cima RFF, Mazurek B, Haider H, Kikidis D, Lapira A, Noreña A, et al. A multidisciplinary European guideline for tinnitus: diagnostics, assessment, and treatment. HNO. (2019) 67:10-42. doi: 10.1007/s00106-019-0633-7

12. Langguth $B$, Landgrebe $M$, Kleinjung $T$, Sand GP, Hajak G. Tinnitus and depression. World J Biol Psychiatry. (2011) 12:489-500. doi: 10.3109/15622975.2011.575178

13. Landgrebe M, Zeman F, Koller M, Eberl Y, Mohr M, Reiter J, et al. The Tinnitus Research Initiative (TRI) database: a new approach for delineation of tinnitus subtypes and generation of predictors for treatment outcome. BMC Med Inform Decis Mak. (2010) 10:42. doi: 10.1186/1472-6947-10-42

14. Luetzenberg FS, Babu S. Alternative treatments of tinnitus : alternative medicine tinnitus alternative medicine treatment. Otolaryngol Clin NA. (2020) 53:637-50. doi: 10.1016/j.otc.2020.03.011

15. Excellence NI for $\mathrm{H}$ and C. Tinnitus: Assessment and Management, NICE guideline (2020). Available online at: https://www.nice.org.uk/ guidance/ng155/resources/tinnitus-assessment-and-management-pdf66141841962949

16. Landry EC, Sandoval XCR, Simeone CN, Tidball G, Lea J, Westerberg BD. Systematic review and network meta-analysis of cognitive and/or behavioral therapies (CBT) for tinnitus. Otol Neurotol. (2020) 41:153-66. doi: 10.1097/MAO.0000000000002472

17. Duckert L, Rees T. Placebo effect in tinnitus management. Otolaryngol Head Neck Surg. (1984) 92:697-9. doi: 10.1177/019459988409200618

18. Hiller W, Goebel G. Factors influencing tinnitus loudness and annoyance. Arch Otolaryngol Head Neck Surg. (2006) 132:1323-30. doi: 10.1001/archotol.132.12.1323 
19. Jastreboff PJ, Jastreboff MM. Decreased Sound Tolerance: Hyperacusis, Misophonia, Diplacousis, and Polyacousis. Vol. 129. 1st ed. Amsterdam: Elsevier BV. (2015). doi: 10.1016/B978-0-444-62630-1.00021-4

Conflict of Interest: MV and HG were employed by Tinnitus Hub Ltd.

The remaining authors declare that the research was conducted in the absence of any commercial or financial relationships that could be construed as a potential conflict of interest.

Publisher's Note: All claims expressed in this article are solely those of the authors and do not necessarily represent those of their affiliated organizations, or those of the publisher, the editors and the reviewers. Any product that may be evaluated in this article, or claim that may be made by its manufacturer, is not guaranteed or endorsed by the publisher.

Copyright (c) 2022 Smit, Vesala, Goedhart, Eijden, Wempe and Stegeman. This is an open-access article distributed under the terms of the Creative Commons Attribution License (CC BY). The use, distribution or reproduction in other forums is permitted, provided the original author $(s)$ and the copyright owner(s) are credited and that the original publication in this journal is cited, in accordance with accepted academic practice. No use, distribution or reproduction is permitted which does not comply with these terms. 